\title{
Integration of Lower Bound Estimates in Pseudo-Boolean Optimization
}

\author{
Vasco M. Manquinho and João Marques-Silva \\ IST/INESC-ID, Technical University of Lisbon, Portugal \\ \{vmm,jpms\}@sat.inesc-id.pt
}

\begin{abstract}
Linear Pseudo-Boolean Optimization (PBO) has found applications in several areas, ranging from Artificial Intelligence to Electronic Design Automation. Due to important advances in Boolean Satisfiability (SAT), new algorithms for PBO have emerged, which are effective on highly constrained instances. However, those algorithms fail in dealing properly with the objective function of PBO. This paper proposes an algorithm that uses lower bound estimation methods for pruning the search tree in integration with techniques from SAT algorithms. Moreover, the paper shows that the utilization of lower bound estimates can dramatically improve the overall performance of $\mathrm{PBO}$ solvers for specific classes of instances. In addition, the paper describes how to apply non-chronological backtracking in the presence of conflicts that result from the bounding process, using different lower bound estimation methods.
\end{abstract}

\section{Introduction}

Recent advances in Boolean Satisfiability (SAT) have resulted in new and effective algorithms for solving the Linear Pseudo-Boolean Optimization problem $[2,4,7]$. These algorithms perform a linear search on the possible values of the cost function, starting from the highest, at each step requiring the next computed solution to have a cost lower than the previous one. If the resulting instance is not satisfiable, then the optimal value is given by the last computed solution. By incorporating important features from SAT solvers like non-chronological backtracking in the search tree, conflict-based learning mechanisms and lazy data structures, these solvers have been able to solve with success several classes of highly constrained pseudo-boolean instances. However, they fail in dealing with the information provided by the cost func- tion. In order to prune the search due to the value of the cost function we propose the use of methods to estimate a lower bound on the value of the cost function. Whenever the lower bound estimation is higher or equal to the best solution found so far, we are able to prune the search tree. Moreover, we also establish conditions for backtracking non-chronologically in the search tree when the search backtracks due to the lower bound estimate.

In this paper we start by describing different lower bound estimation methods for the pseudo-boolean optimization problem, focusing on linear-programming relaxation and Lagrangian relaxation. In section 4 we describe how to obtain explanations on bound-conflict situations that allow backtracking non-chronologically when the search is bound due to the lower bound estimate. We also address how the results from the lower bound methods can be used to guide the search. Finally, we present some experimental results and the paper concludes in section 7 .

\section{Preliminaries}

In a propositional formula, a literal $l_{j}$ denotes either a variable $x_{j}$ or its complement $\bar{x}_{j}$. If a literal $l_{j}=x_{j}$ and $x_{j}$ is assigned value 1 or $l_{j}=\bar{x}_{j}$ and $x_{j}$ is assigned value 0 , then the literal is said to be true. Otherwise, the literal is said to be false.

An instance $P$ of a Linear Pseudo-Boolean Optimization problem can be defined as follows,

$$
\begin{array}{ll}
\text { minimize } & \sum_{j=1}^{n} c_{j} \cdot x_{j} \\
\text { subject to } & \sum_{j=1}^{n} a_{i j} l_{j} \geq b_{i}, \\
& x_{j} \in\{0,1\}, \\
& a_{i j}, b_{i} \in N_{0}^{+}, i \in\{1 . . m\}
\end{array}
$$

where $c_{j}$ is a non-negative integer cost associated with variable $x_{j}, 1 \leq j \leq n$ and $a_{i j}$ denote the coefficients 
of the literals $l_{j}$ in the set of $m$ linear constraints. Every pseudo-boolean formulation can be rewritten such that all coefficients $a_{i j}$ and right-hand side $b_{i}$ be nonnegative.

In a given constraint, if all $a_{i j}$ coefficients have the same value $k$, then it is called a cardinality constraint, since it only requires that $\left\lceil b_{i} / k\right\rceil$ literals be true. A pseudo-boolean constraint where any literal set to true is enough to satisfy the constraint, can be interpreted as a propositional clause. This occurs when the value of all $a_{i j}$ coefficients are greater than or equal to $b_{i}$.

If every constraint can be interpreted as a propositional clause then $P$ is an instance of the binate covering problem (BCP). Covering formulations have been the subject of thorough research work that can be found in $[5,11,17]$.

Notice that a linear pseudo-boolean optimization problem can also be viewed as a special case of linear integer programming problem. The linear integer programming formulation for the constraints can be obtained if we replace literals $\bar{x}_{j}$ by $1-x_{j}$. In section 3 we will use this latter formulation.

\section{Pseudo-Boolean Optimization Algo- rithms}

In [3], P. Barth first proposed an approach based on Boolean Satisfiability (SAT) techniques for solving Pseudo-Boolean Optimization (PBO). This approach consists of performing a linear search on the possible values of the cost function, starting from the highest, at each step requiring the next computed solution to have a cost lower than the previous one. If the resulting instance is not satisfiable, then the solution is given by the last recorded solution. The generalization of recent advances in SAT resulted in new successful algorithms $[2,4,7]$ for several sets of PBO instances, namely the incorporation of non-chronological backtracking in the search tree, conflict-based learning mechanisms and lazy data structures have been applied with success. The SAT-based approach focuses primarily on finding solutions for the problem constraints. Therefore, for highly constrained problems these techniques are very effective. However, these algorithms find it difficult to deal with the information from the cost function.

Unlike the SAT-based approach, branch-and-bound algorithms [6, 9] have proved to be very effective when the instances to be solved are not highly constrained since they are able to prune the search tree earlier due to estimate of the value of the cost function. In branchand-bound algorithms upper bounds on the value of the cost function are identified for each solution to the con- straints, and lower bounds on the value of the cost function are estimated considering the current set of variable assignments. For a given instance $P$ of a pseudoboolean optimization problem, let P.upper denote the upper bound on the value of the cost function. The search is pruned whenever the lower bound estimation is higher than or equal to P.upper. In this case it is guaranteed that a better solution cannot be found with the current variable assignments and therefore the search can be pruned. The algorithms described in $[5,9,11,17]$ for the binate covering problem follow this approach as well as several general integer programming solvers.

For several instances, specially for low constrained instances, the tightness of the lower bounding procedure is crucial for the algorithm's efficiency, because with higher estimates of the lower bound, the search can be pruned earlier. Several procedures can be used for lower bound estimation, namely the approximation of a maximum independent set of constraints (MIS) [6, 11], linear-programming relaxations [9] or Lagrangian relaxations [14].

\subsection{Linear Programming Relaxations}

Although the approximation of a maximum independent set of constraints (MIS) is the most widely used lower bound procedure for the binate covering problem (a particular case of PBO) [5, 17], linear programming relaxation (LPR) has also been used with success [9] . It is also often the case that the linear programming relaxation bound is higher than the one obtained with the MIS approach. Nevertheless, linear programming relaxations have long been used as a lower bound estimation procedure in branch-and-bound algorithms for solving integer programming problems [13].

The general formulation of the LPR for a pseudoboolean problem is obtained from (1) as follows:

$$
\begin{array}{ll}
\text { minimize } & z_{l p r}=c x \\
\text { subject to } & A x \geq b \\
& x \geq 0
\end{array}
$$

where vector $c$ defines the non-negative integer cost associated with every decision variable in vector $x$. Entries of matrix $A$ defines the constraint coefficients and vector $b$ the right-hand side of every constraint. For simplicity the constraints $x \leq 1$ are not included. The solution of (1) is referred to as $z_{c p}^{*}$, whereas the solution of (2) is referred to as $z_{l p r}^{*}$.

It is well-known that the solution $z_{l p r}^{*}$ of (2) is a lower bound on the solution $z_{c p}^{*}$ of (1) [13]. Basically, any solution of (1) is also a feasible solution of (2), but the converse is not true. Moreover, for a given solution of (2) where $x \in\{0,1\}^{n}$, we necessarily have 
$z_{c p}^{*}=z_{l p r}^{*}$. Hence, the result follows. Furthermore, different linear programming algorithms can be used for solving (2), some of which with guaranteed worst-case polynomial run time [13].

\subsection{Lagrangian Relaxations}

Lagrangian relaxation (LGR) is a widely used method for computing bounds on the optimal value of the cost function from network optimization to nonlinear programming $[14,15]$. It also known that in some instances, the bound provided by the Lagrangian relaxation method is tighter than the one obtained by the linear programming relaxation [14]. Therefore, Lagrangian relaxation can be used to provide a quick and tight lower bound on the value of the cost function for pseudo-boolean optimization problems.

While in linear programming relaxations we are able to find a lower bound estimate by solving the problem constraints and relaxing the possible variable values, in Lagrangian relaxations we relax the problem constraints and incorporate them in the objective function with associated Lagrangian multipliers.

Given a generic linear optimization problem formulated as:

$$
\begin{array}{ll}
\operatorname{minimize} & z^{*}=c x \\
\text { subject to } & A x=b \\
& x \in X
\end{array}
$$

we can define the Lagrangian function $L(\mu)$ as:

$$
L(\mu)=\min \{c x+\mu(A x-b): x \in X\}
$$

where vector $\mu$ defines the Lagrangian multiplier associated with each constraint. The Lagrangian Bounding Principle [14] states that for any vector $\mu$ of the Lagrangian multipliers, the value of $L(\mu)$ is a lower bound on the optimal solution of the original optimization problem.

In (3) all constraints are formulated as equalities, while in the pseudo-boolean optimization problem (1) we have inequality constraints. Therefore, in that case the Lagrangian relaxation problem is formulated as:

$$
L^{*}=\max \{L(\mu): \mu \geq 0\}
$$

where $L^{*}$ is the optimum value of the Lagrangian relaxation. The most tight lower bound estimate we can obtain using this method is given by $\left\lceil L^{*}\right\rceil$.

Before trying to solve the Lagrangian relaxation problem in order to obtain $L^{*}$, we must determine the value of $L(\mu)$ for a given value of $\mu$. Notice that by ex- panding (4) we have:

$$
\begin{aligned}
& L(\mu)=\min \left\{\sum_{j=1}^{n} c_{j} x_{j}+\sum_{i=1}^{m} \mu_{i}\left(\left(\sum_{j=1}^{n} a_{i j} x_{j}\right)-b_{i}\right)\right\} \\
& L(\mu)=\min \left\{\sum_{j=1}^{n} c_{j} x_{j}+\sum_{i=1}^{m} \mu_{i}\left(\sum_{j=1}^{n} a_{i j} x_{j}\right)-\sum_{i=1}^{m} \mu_{i} b_{i}\right\} \\
& \left.L(\mu)=\min \left\{\sum_{j=1}^{n}\left(c_{j}+\sum_{i=1}^{m} \mu_{i} a_{i j}\right) x_{j}\right)-\sum_{i=1}^{m} \mu_{i} b_{i}\right\} \\
& L(\mu)=\min \left\{\sum_{j=1}^{n} \alpha_{j} x_{j}-\sum_{i=1}^{m} \mu_{i} b_{i}\right\} \text { where } \\
& \alpha_{j}=\left(c_{j}+\sum_{i=1}^{m} \mu_{i} a_{i j}\right)
\end{aligned}
$$

In order to obtain the value of $L(\mu)$, for a given $\mu$, we must determine the value of the decision variables $x_{j}$ to be able to minimize the expression. Therefore, we must have $x_{j}=0$ whenever $\alpha_{j} \geq 0$ and $x_{j}=1$ when $\alpha_{j}<0$.

Literature from nonlinear programming [15] and network optimization [14] provide methods to solve (5), namely gradient methods to approximate the value of $L^{*}$. Let $\mu^{k}$ be the value of the Lagrangian multipliers at iteration $k . \mu^{0}$ can be any choice of the values of the Lagrangian multipliers ( 0 was our arbitrarily option). In the next iterations, the value of the Lagrangian multipliers is updated according to:

$$
\mu^{k+1}=\max \left\{0, \mu^{k}+\theta^{k}\left(A x^{k}-b\right)\right\}
$$

where $\theta^{k}$ is the gradient step at iteration $k$. Remember that since we are dealing with inequality constraints, the values of the Lagrangian multipliers cannot be negative. Several heuristics can be used in order to define the value of $\theta^{k}$ at each iteration such that it guarantees the convergence of the algorithm. One widely used heuristic [14] is defined as follows:

$$
\theta^{k+1}=\frac{\lambda^{k}\left[U B-L\left(\mu^{k}\right)\right]}{\left\|\left(A x^{k}-b\right)\right\|^{2}}
$$

where $U B$ is an upper bound on the optimal value of the pseudo-boolean problem and $\lambda^{k}$ is a value chosen between 0 and 2. Usually we have that $\lambda^{0}=2$. If in a given number of iterations the best value for the Lagrangian function does not improve, then the value of $\lambda$ is reduced by a factor of 2 .

Notice that at a given iteration $k$, if the assignments made to the decision variables in $x^{k}$ satisfy all problem constraints and P.upper $>L\left(\mu^{k}\right)$, then the upper bound is updated. However, we can only bound the search when the optimum value of the Lagrangian relaxation $L^{*}$ is higher than or equal to the upper bound 
P.upper. This situation occurs when the assignments to decision variables in $x^{k}$ satisfy all problem constraints and $\mu^{k}\left(A x^{k}-b\right)=0$ and $L\left(\mu^{k}\right)$ denotes the optimum value of the Lagrangian relaxation $L^{*}$. Therefore, the gradient method is stopped, the upper bound is updated if P.upper $>L^{*}$ and we bound the search.

In most cases, the convergence of the gradient method can be quite slow. If we are unable to improve on the best value for the Lagrangian function in a given number of iterations or if a limit number of iterations is reached, we stop the Lagrangian function computation and continue the search. Another stopping criteria is when the value of $\left\lceil L\left(\mu^{k}\right)\right\rceil$ is higher than or equal to the value of the best solution of the original problem we found so far. In this case we have a bound conflict, the Lagrangian function computation is stopped and we bound the search.

\section{Bound-based Conflicts}

In [11] is proposed a framework to backtrack nonchronologically due to the lower bound estimate on the value of the cost function. In this section we review the main ideas about pruning the search tree based on the estimated value of the cost function and describe the conditions when using linear-programming relaxation or Lagrangian relaxation as a lower bound estimation procedure.

\subsection{Backtracking on Bound-based Con- flicts}

A bound conflict in an instance of the pseudoboolean optimization problem $(\mathrm{PBO}) P$ arises when the lower bound is equal to or higher than the upper bound. This condition can be written as

$$
\text { P.path }+ \text { P.lower } \geq \text { P.upper }
$$

where P.path is the cost of the assignments already made, $P$.lower is a lower bound estimate on the cost of satisfying the constraints not yet satisfied (as given for example using Lagrangian relaxation), and P.upper is the best solution found so far.

In this situation, our approach is to identify a set of assignments responsible for the bound conflict and build a new propositional clause $\omega_{b c}$ such that it prevents those assignments of being repeated during the search process. When $\omega_{b c}$ is added, a conflict analysis procedure must be carried out to determine to which level of the search tree to backtrack to.

A straightforward approach to build $\omega_{b c}$ would be to consider the decision variable assignments from all lev- els of the search tree, but in that case the resulting backtrack would necessarily be chronological. In [11] it was already shown that the assignments responsible for the bound conflict might not be associated with all levels of the search tree.

From (9), we can readily conclude that P.path and $P$.lower are the unique components involved in each bound conflict. Therefore, we will analyze both the P.path and P.lower components in order to establish the assignments responsible for a given bound conflict. Our goal is to define two sets of literals $\omega_{p p}$ and $\omega_{p l}$ containing the explanation for P.path and P.lower, respectively. Our bound conflict clause $\omega_{b c}$ is defined by the set union of the literals in $\omega_{p p}$ and $\omega_{p l}$.

We start by studying P.path. Clearly, the variable assignments that cause the value of P.path to grow are solely those assignments with a value of 1 . Hence, we can define $\omega_{c p}$ such that each variable in $\omega_{c p}$ has positive cost and is assigned value 1 :

$$
\omega_{p p}=\left\{l=\bar{x}_{j}: \operatorname{Cost}\left(x_{j}\right)>0 \wedge x_{j}=1\right\}
$$

which basically states that in order to decrease the value of the cost function (i.e.P.path) at least one variable that is assigned value 1 has instead to be assigned value 0 .

We now consider P.lower. Since different lower bound estimation procedures can be used, we will describe in the remainder of this section how to identify an explanation for the bound conflict when using either linear-programming relaxation or Lagrangian relaxation.

\subsection{Lower Bound Conflicts from Linear- Programming Relaxation}

When using linear-programming relaxations as a lower bound estimation procedure, the value of P.lower is obtained according to the formulation described in section 3.1. In order to determine the set of assignments we can deem responsible for P.lower, we must define $S$ as the set of constraints with slack ${ }^{1}$ variables assigned value 0 in the linear program solution. These are the constraints which actually limit the value of P.lower. If the literals that assume value 0 in these constraints were to have a different value, some constraints might be satisfied and the value of P.lower would be lower. Therefore, we can consider the assignments to those literals as the responsible for P.lower and define $\omega_{p l}$ as:

$$
\omega_{p l}=\left\{l: l=0 \wedge l \in \omega_{i} \wedge \omega_{i} \in S\right\}
$$

1 See [10] for a definition of slack and artificial variables. 
Clearly, $\omega_{p l}$ does not necessarily depend on all decision levels in the search tree. Hence, non-chronological backtracking might result from the conflict analysis procedure.

\subsection{Lower Bound Conflicts from La- grangian Relaxation}

In order to determine $\omega_{p l}$ using the Lagrangian relaxation lower bound estimation procedure as described in section 3.2, we can follow a similar approach to the one described for linear-programming relaxation. Let $S$ be the set of constraints used in obtaining the value of P.lower whose Lagrangian multiplier is different from 0 . We can clearly notice from (6) that the constraints with Lagrangian multiplier equal to 0 are irrelevant for computing P.lower. In this case, $\omega_{p l}$ can be determined as formulated in (11).

Another approach to determine $\omega_{p l}$ is to consider the value of $\alpha_{j}$ for each assigned variable from $S$. If a given variable $x_{j}$ is assigned value 0 and $\alpha_{j}>0$, then by changing its value to 1 we would increase the value of P.lower. Or if variable $x_{j}$ is assigned value 1 and $\alpha_{j}<0$, if we were to change the value of $x_{j}$, P.lower would raise. Hence, these assignments cannot be deemed responsible for the value of P.lower and should not be considered in $\omega_{p l}$.

\section{Heuristics}

Almost all current SAT solvers use the VSIDS [12] heuristic of variation thereof since it is commonly accepted that the variable decision assignment should be quick while most effort should be spent on boolean constraint propagation and efficient conflict analysis strategies for backtracking. Therefore, the VSIDS heuristic is based on a very simple concept. For each literal there is a counter and whenever a new conflict-induced clause is added, the counters of the literals in the new clause are incremented. The decision variable assignment selection simply chooses the assignment that satisfy the literal with the highest counter value. New SAT-based pseudo-boolean solvers $[2,4,7]$ also follow this approach.

However, one should note that the lower bound methods described in section 3 already provide a possible guide to satisfy the problem constraints while minimizing the value of the cost function. We propose to take advantage of the computation effort already spent in the lower bound estimation for a more informed decision variable assignment selection without an additional overhead to the process. Our proposal is to se-

\begin{tabular}{llrrr}
\hline & \multicolumn{4}{c}{ no explanations } \\
\hline Benchmark & Sol. & CPU & \#Dec. & \#NCB \\
\hline 9symml & 4517 & ub 4875 & 130213 & 1 \\
C17 & 260 & 0.00 & 0 & 0 \\
C432 & 4822 & ub 4860 & 157299 & 5 \\
b1 & 128 & 0.00 & 0 & 0 \\
c8 & 1194 & 0.91 & 252 & 0 \\
cc & 1567 & 0.08 & 97 & 0 \\
cm42a & 694 & 0.04 & 9 & 0 \\
cmb & 1053 & 25.98 & 3806 & 0 \\
mux & 872 & 0.06 & 0 & 0 \\
my_adder & 4561 & 8.12 & 1384 & 2 \\
\hline
\end{tabular}

Table 1. Using Lagrangian relaxation

lect the variable assignment that satisfies the literal with the highest counter value, but only considering the assignments made in finding the best lower bound estimated value.

\section{Experimental Results}

In this section we present empirical results for the techniques described in the paper using our pseudoboolean optimizer (bsolo) which incorporates classical branch-and-bound techniques described in the paper and SAT-based techniques, namely boolean constraint propagation, non-chronological backtracking in the search tree and conflict-based learning mechanisms.

The CPU times presented are from a AMD Athlon processor at $1.9 \mathrm{GHz}$ with $1 \mathrm{~GB}$ of physical memory. The time limit for each instance was set to one hour. If the time limit was reached, we provide an indication of which was the best upper bound value found when the search was stopped.

In order to empirically test the lower bound procedures described in the paper, we ran our solver in a benchmark set from a problem of synthesis for mixed PTL/CMOS circuits from [18]. Although, these benchmarks are mainly instances from the binate covering problem they provide a good insight on the efficiency of these techniques. We also present results using the routing benchmarks from [1] with the objective of trying to minimize the number of variables assigned value 1.

Our solver was configured to use the constraint strengthening technique described in [7] and widely used in mixed integer programming [16]. The probing used in the constraint strengthening is also used to detect necessary assignments during preprocessing. We also used simplification techniques described in $[8,17]$ in the synthesis benchmark set. 


\begin{tabular}{llrrr}
\hline & \multicolumn{4}{c}{ using explanations } \\
\hline Benchmark & Sol. & CPU & \#Dec. & \#NCB \\
\hline 9symml & 4517 & ub 4720 & 194706 & 32671 \\
C17 & 260 & 0.00 & 0 & 0 \\
C432 & 4822 & 1174.40 & 130874 & 15222 \\
b1 & 128 & 0.00 & 0 & 0 \\
c8 & 1194 & 1.58 & 309 & 39 \\
cc & 1567 & 0.39 & 357 & 28 \\
cm42a & 694 & 0.04 & 9 & 0 \\
cmb & 1053 & 12.16 & 2247 & 401 \\
mux & 872 & 0.06 & 0 & 0 \\
my_adder & 4561 & 7.57 & 1307 & 100 \\
\hline
\end{tabular}

Table 2. Using Lagrangian relaxation

\begin{tabular}{lrrrr}
\hline & \multicolumn{2}{c}{ LP-relax. } & \multicolumn{2}{c}{ LGR-relax. } \\
\hline Bench. & CPU & \#Dec. & CPU & \#Dec. \\
\hline 9symml & ub 4517 & 330133 & 347.13 & 6921 \\
C17 & 0.00 & 0 & 0.00 & 0 \\
C432 & ub 4895 & 487461 & 279.25 & 3462 \\
b1 & 0.00 & 0 & 0.00 & 0 \\
c8 & 0.49 & 100 & 0.19 & 14 \\
cc & 0.13 & 51 & 0.09 & 49 \\
cm42a & 0.11 & 44 & 0.06 & 17 \\
cmb & 34.70 & 3904 & 0.43 & 21 \\
mux & 0.00 & 0 & 0.00 & 0 \\
my_adder & 591.24 & 60814 & 0.77 & 54 \\
\hline
\end{tabular}

Table 3. Heuristic proposed in section 5

In Tables 1 and 2 we present our results for our pseudo-boolean solver using VSIDS heuristic and Lagrangian relaxation as the lower bound method. In this table we provide the CPU time in seconds, the number of decisions and the number of non-chronological backtracks observed during the search, and evaluate the use of explanations described in section 4.3 when bound conflicts occur. Notice that there is a significant reduction of the search space and time spent in solving these instances.

In Table 3 we can see the results for this benchmark set when using the heuristic proposed in section 5 . Linear-programming relaxation provides good results, but Lagrangian relaxation is able to solve all instances with dramatically better CPU times.

In comparison with the SAT-based linear search algorithms $[2,3,4,7]$, our approach is able to perform much better due to the lower bounding procedures incorporated in our algorithm. All other algorithms find it very difficult to deal with the information from the cost function and are able to solve just the more easy problem instances, as we can see in table 4 . In most in-

\begin{tabular}{llrrr}
\hline & & PBS & galena & bsolo \\
\hline Benchmark & Sol. & CPU & CPU & CPU \\
\hline 9symml & 4517 & ub 6453 & ub 6986 & 347.13 \\
C17 & 260 & 0.00 & 0.01 & 0.00 \\
C432 & 4822 & ub 6577 & ub 8070 & 279.25 \\
b1 & 128 & 0.00 & 0.00 & 0.00 \\
c8 & 1194 & ub 1542 & ub 1528 & 0.19 \\
cc & 1567 & ub 1692 & ub 1786 & 0.09 \\
cm42a & 694 & ub 754 & ub 696 & 0.06 \\
cmb & 1053 & ub 1490 & ub 1476 & 0.43 \\
mux & 872 & ub 1321 & ub 1333 & 0.00 \\
my_adder & 4561 & ub 6271 & ub 5548 & 0.77 \\
\hline
\end{tabular}

Table 4. Comparison with SAT-based algorithms

\begin{tabular}{llrrr}
\hline & & PBS & galena1 & galena2 \\
\hline Benchmark & Sol. & CPU & CPU & CPU \\
\hline grout-4.3.1 & 62 & ub 64 & ub 62 & 2.85 \\
grout-4.3.2 & 64 & ub 66 & 594.37 & 19.42 \\
grout-4.3.3 & 62 & ub 66 & ub 62 & 4.11 \\
grout-4.3.4 & 60 & ub 62 & ub 60 & 6.51 \\
grout-4.3.5 & 60 & ub 64 & 1373.29 & 6.02 \\
grout-4.3.6 & 66 & 617.61 & 58.93 & 8.26 \\
grout-4.3.7 & 64 & 1334.94 & 50.23 & 0.86 \\
grout-4.3.8 & 36 & ub 44 & ub 42 & 17.90 \\
grout-4.3.9 & 68 & 1227.29 & 150.03 & 2.24 \\
grout-4.3.10 & 70 & 36.16 & 41.09 & 0.36 \\
\hline
\end{tabular}

Table 5. Comparison in routing benchmarks

stances galena [4] was unable to finish due to memory constraints after several minutes running while $P B S[2]$ was unable to solve these instances due to time limits.

Finally, we present the results for the routing benchmarks. galena1 means that the selected learning scheme was the generation of a propositional clause (like $P B S$ and bsolo) while in galena2 the selected learning scheme was the generation of a cardinality constraint (default option of the solver). We also present two columns regarding bsolo. In bsolo1 linear-programming relaxations are used while in bsolo2 we used Lagrangian relaxations.

We can see that for these instances linearprogramming relaxation performed better than Lagrangian relaxation. A more detailed analysis revealed that Lagrangian relaxation needs more iterations in order to produce a tighter lower bound estimation than for the synthesis benchmark set. One should also notice the very effective behavior of galena when learning cardinality constraints from the conflict analysis procedure, enabling galena to solve all instances in 


\begin{tabular}{llrr}
\hline & & bsolo1 & bsolo2 \\
\hline Benchmark & Sol. & CPU & CPU \\
\hline grout-4.3.1 & 62 & 0.27 & ub 64 \\
grout-4.3.2 & 64 & 1139.80 & ub 64 \\
grout-4.3.3 & 62 & ub 66 & ub 66 \\
grout-4.3.4 & 60 & 1942.10 & ub 60 \\
grout-4.3.5 & 60 & 77.86 & 2316.90 \\
grout-4.3.6 & 66 & 2183.30 & 832.94 \\
grout-4.3.7 & 64 & 99.60 & 2777.80 \\
grout-4.3.8 & 36 & 5.23 & 37.23 \\
grout-4.3.9 & 68 & 412.03 & 204.32 \\
grout-4.3.10 & 70 & 0.40 & 5.42 \\
\hline
\end{tabular}

Table 6 . Comparison in routing benchmarks

this benchmark set. These results motivate integrating cardinality constraints learning in bsolo and the development of techniques for fine-tuning Lagrangian relaxation lower bounding, and for dynamically switching between the two lower bound techniques.

\section{Conclusions}

The paper proposes the integration of lower bound estimation procedures with SAT-based techniques for linear pseudo-boolean optimization. We focus mainly in two lower bound methods: linear-programming relaxation and Lagrangian relaxation. We describe a procedure to enable non-chronological backtracking in the search tree when bound conflicts occur. In addition, we also propose the use of information from the lower bound procedure in order to have a more informed heuristic for decision assignment without overhead in the process.

Preliminary results show that for specific classes of instances the integration of lower bound estimation procedures offer a dramatic improvement with respect to linear pseudo-boolean solvers. Results also show that linear search SAT-based algorithms find it very difficult to solve instances when the range of the cost value of feasible solutions is large. When that occurs, the algorithm needs a lower bound estimation procedure that provides a tight bound.

Future research work will include improvements on the integration of the lower bound estimation procedures as well as the development of different conflictbased learning schemes and improve on the implementation of the data structures.

\section{References}

[1] F. Aloul, A. Ramani, I. Markov, and K. Sakallah. Generic ILP versus specialized 0-1 ILP: An update. In IEEE/ACM International Conference on Computer Aided Design, pages pp. 450-457, November 2002.

[2] F. Aloul, A. Ramani, I. Markov, and K. Sakallah. PBS: A Backtrack-Search Pseudo-Boolean Solver. In Symposium on the Theory and Applications of Satisfiability Testing (SAT), pages pp. 346-353, 2002.

[3] P. Barth. A Davis-Putnam Enumeration Algorithm for Linear Pseudo-Boolean Optimization. Technical Report MPI-I-95-2-003, Max Plank Institute for Computer Science, 1995.

[4] D. Chai and A. Kuehlmann. A Fast Pseudo-Boolean Constraint Solver. In Design Automation Conference, 2003.

[5] O. Coudert. On Solving Covering Problems. In Proceedings of the ACM/IEEE Design Automation Conference, pages 197-202, June 1996.

[6] O. Coudert and J. C. Madre. New Ideas for Solving Covering Problems. In Proceedings of the ACM/IEEE Design Automation Conference, June 1995.

[7] H. Dixon and M. Ginsberg. Inference Methods for a Pseudo-Boolean Satisfiability Solver. In National Conference on Artificial Intelligence, 2002.

[8] J. Hooker. Logic-Based Methods for Optimization. In . Jon Wiley \& Sons, 1996.

[9] S. Liao and S. Devadas. Solving Covering Problems Using LPR-Based Lower Bounds. In Proceedings of the ACM/IEEE Design Automation Conference, pages 117$120,1997$.

[10] J. J. J. M. S. Bazaraa and H. D. Sherali. Linear Programming and Network Flows. 2nd Ed., John Wiley \& Sons, 1989.

[11] V. Manquinho and J. Marques-Silva. Search pruning techniques in sat-based branch-and-bound algorithms for the binate covering problem. IEEE Transactions on Computer-Aided Design, 2002.

[12] M. Moskewicz, C. Madigan, Y. Zhao, L. Zhang, and S. Malik. Chaff: Engineering an Efficient SAT Solver. In Design Automation Conference, June 2001.

[13] G. L. Nemhauser and L. A. Wolsey. Integer and Combinatorial Optimization. John Wiley \& Sons, 1988.

[14] T. M. R. Ahuja and J. Orlin. Network Flows: Theory, Algorithms, and Applications. Pearson Education, 1993.

[15] A. S. S. Nash. Linear and Nonlinear Programming. In . McGraw-Hill, 1996.

[16] M. Savelsbergh. Preprocessing and probing for mixed integer programming problems. ORSA Journal on Computing, 6:445-454, 1994.

[17] T. Villa, T. Kam, R. K. Brayton, and A. L. SangiovanniVincentelli. Explicit and Implicit Algorithms for Binate Covering Problems. IEEE Transactions on Computer Aided Design, vol. 16(7):677-691, July 1997.

[18] Z. Zhu. Synthesis for mixed ptl/cmos circuit. http://www-unix.ecs.umass.edu zzhu/. 